\title{
Optimizing the Path Traversed using Artificial Bee Colony Algorithm
}

\author{
Devesh Batra \\ Student, Stanford University, USA
}

\author{
Pragya Verma \\ Member, IEEE
}

\begin{abstract}
With the need of traversing a specified path in shortest time increases the demand of optimizing the route traversed. This optimization involves path or trajectory planning along with the implementation of an optimization algorithm. Several Swarm Intelligence techniques have been applied to solve the optimization problems. In this paper, we discuss the optimization achieved with the usage of one of the Swarm Intelligence algorithms namely, Artificial Bee colony Optimization. Implementation of Artificial Bee Colony Optimization helps in finding the shortest, collision-free path from a specified starting point to the predetermined destination or goal point with consideration to static or dynamic obstacles.
\end{abstract}

\section{Keywords}

Path Planning, Swarm Intelligence, Artificial Bee Colony Optimization, Static and Dynamic Obstacles, Obstacle Detection, Obstacle Avoidance

\section{INTRODUCTION}

Path Optimization can be defined as traversing the path in the shortest or the most optimum manner. There are times, when the shortest path is not the most optimum path from a starting node to a predefined destination or goal point due to presence of unavoidable obstacles and/or congestion. In such cases, the path which should be chosen should be the one in which the minimum time is taken in order to complete the journey.

Many day to day applications require the most optimum path to be traversed. Travelling salesman problem is a very famous problem which has path optimization as its requirement. Thus, the implementation of several path optimization techniques can be extended to many real world, day to day applications.

Computer scientists have drawn inspiration from the behaviour of swarms. Their complex problem solving skills and path optimization techniques not only fascinates the biologists but also the computer scientists. Swarm Intelligence can be defined as a field of computer science that designs and studies efficient computational method for solving problems in a way that is inspired by the behaviour of real swarms or insect colonies [1].

Three components play an important role in the implementation and development of Swarm Intelligence techniques. These three things are as follows - Evaluation, Comparison and Imitation.

Evaluation can be defined as estimation of the behaviour of several swarm species or insects. Comparison is the process of drawing an analogy between the way swarm species behave and human behaviour. Imitation is an effective form of learning It is the process in which swarm behaviour is cloned and applied to day to day activities of humans [2].

Path planning can be defined as the process of converting high level specification of task into low level determination of how to move. The goal of path planning is to reach the goal or destination point as fast as possible with avoidance of obstacles encountered while traversing the path.

The paper is presented as follows: section II consists of review of literature. Section III describes the concepts of Swarm Intelligence and Artificial Bee Colony Algorithm which is to be used in order to achieve path optimization. Section IV presents a comparison between Ant Colony Algorithm and Artificial Bee Colony Algorithm for path optimization. Section $\mathrm{V}$ presents the conclusions of the findings presented in this paper.

\section{REVIEW OF LITERATURE}

[3] Presents a metaheuristics algorithm for discrete optimization that that has taken inspiration from the foraging behaviour of ants, their colonies. It is observed that Ant Colony Optimization is promising technique and metaheuristics ACO is a new wave of stochastic metaheuristics such as evolutionary computation, simulated annealing, tabu search and neural computation.

In [4] a detailed description of Firefly Algorithm for multimodal optimization applications is presented. With the help of simulations and results it has been observed that the proposed Firefly Algorithm is better than the many existing metaheuristics algorithms.

In [5] an application of particle swarm optimization for travelling salesman problem is presented. Further, the concept of swap operator and swap sequence is proposed. The results obtained are promising.

A cooperative learning approach to complex transportation problems is presented in [6]. In this paper, a bee colony optimization metaheuristics (BCO) is proposed. Further, it is observed that $\mathrm{BCO}$ is capable of solving several problems ranging from deterministic combinatorial problems to combinatorial problems characterized by uncertainty.

\section{THEORETICAL BACKGROUND 3.1 Swarm Intelligence}

Swarm Intelligence can be defined as a field of computer Science that designs and studies efficient computational method for solving complex problems in a way inspired by the behaviour of real swarms or insect colonies [1].

Few eminent properties of swarm intelligence can be defined as follows:

1. Swarm consists of several individuals

2. These several individuals are homogenous genetically.

3. The interaction between individuals is based on local information 
Five fundamental principles play an important role in defining swarm intelligence. These five principles can be stated as follows:

1. Proximity Principle: This principle states that the individuals should be able to carry out simple time and space computations.

2. Quality Principle: This principle states that the individual should be able to respond effectively to the quality factors in the environment.

3. Diverse Response Principle: According to this principle the individual should not commit its activity along excessively narrow channels

4. Stability Principle: According to this principle the individual should never change its mode of behaviour with the change in the environment.

5. Adaptability Principle: It should be easy for the individual to change its behaviour mode when the computational price is worth the change.

Swarm Intelligence can be successfully applied to various applications such as combinatorial optimization problems, function optimization, finding optimal routes, scheduling, structural optimization, image analysis, data mining, machine learning, bioinformatics, medical informatics, dynamic systems, industrial problems and at times even at finance and business [7]. It has been observed that Swarm Intelligence based techniques are more effective for dynamic problems than for the static ones that do not change over time.

Scalability, Adaptability, Collective Robustness and Individual Simplicity are some of the advantages of Swarm Intelligence based algorithms. These advantages make the Swarm Intelligence based techniques more efficient than the traditional optimization techniques.

\subsection{Artificial Bee Colony Algorithm}

Artificial Bee Colony Algorithm (ABC) is motivated by the intelligent behaviour of the bees. Colony size and maximum cycle number are examples of common control parameters.

In $\mathrm{ABC}$, a population based search procedure is used. In this procedure, individuals called food positions are changed by the artificial bees and the main goal of the bee is to discover the places food sources with high amount of nectar and finally the one with the highest amount of nectar [8].

In such a system, artificial bees fly around in a multidimensional search space and the employed and the onlooker bees choose food sources depending on their own experiences and their nest mates and thus adjust their positioning.

Whereas the Scout Bees fly and select the food sources randomly without using any kind of experience. If the amount of nectar a new source is greater than that of the one found previously in their memory, they learn this new position and forget the previous one.

Thus, ABC combines local search methods, carried out by employed and onlooker bees, with the global search methods, managed by onlooker and scout bees, thereby balancing exploration and exploitation process.

An artificial onlooker bee chooses a food source depending on the probability value associated with that food source, pi , calculated by the following expression:

$$
p_{i}=\frac{f_{i t_{i}}}{\sum_{n=1}^{S N} f_{i t_{n}}}
$$

where fiti is the fitness value of the solution $i$ which is proportional to the nectar amount of the food source in the position $\mathrm{i}$ and $\mathrm{SN}$ is the number of food sources which is equal to the number of employed bees (BN) [9].

In order to produce a candidate food position from the old one in memory, the $\mathrm{ABC}$ uses the following expression:

$$
v_{i j}=x_{i j}+\phi_{i j}\left(x_{i j}-x_{k j}\right)
$$

where $\mathrm{k} \in\{1,2, \ldots, \mathrm{SN}\}$ and $\mathrm{j} \in\{1,2, \ldots, \mathrm{D}\}$ are randomly chosen indexes. Although $\mathrm{k}$ is determined randomly, it has to be different from i. $\varphi \mathrm{i}, \mathrm{j}$ is a random number between $[-1,1]$. It controls the production of neighbour food sources around $x i, j$ and represents the comparison of two food positions visually by a bee.

If a parameter value produced by this operation exceeds its predetermined limit, the parameter can be set to an acceptable value. In this work, the value of the parameter exceeding its limit is set to its limit value.

The food source whose nectar is deserted by the bees is replaced with a new food source by the scouts. In ABC, this is simulated by producing a position randomly and replacing it with the abandoned one. In $\mathrm{ABC}$, providing that a position cannot be improved further through a predetermined number of cycles, then that food source is assumed to be abandoned. The value of predetermined number of cycles is an important control parameter of the $\mathrm{ABC}$ algorithm, which is called "limit" for abandonment. Assume that the abandoned source is $x i$ and $j \in\{1,2, \ldots, D\}$, then the scout discovers a new food source to be replaced with xi. This operation can be defined as

$$
x_{i}^{j}=x_{\min }^{j}+\operatorname{rand}(0,1)\left(x_{\max }^{j}-x_{\min }^{j}\right)
$$

After each candidate source position vi,j is produced and then evaluated by the artificial bee, its performance is compared with that of its old one. If the new food has equal or better nectar than the old source, it is replaced with the old one in the memory. Otherwise, the old one is retained in the memory. In other words, a greedy selection mechanism is employed as the selection operation between the old and the candidate one.

\subsection{Pseudo Code for Artificial Bee Colony Algorithm}

1. Initialize the population of solutions $x i, j, i=1 \ldots S N, j=$ 1. . . D

2. Evaluate the population

3. cycle $=1$

4. repeat

5. Produce new solutions vi,j for the employed bees by using (2) and evaluate them

6. Apply the greedy selection process

7. Calculate the probability values $\mathrm{Pi}, \mathrm{j}$ for the solutions $\mathrm{xi}, \mathrm{j}$ by (1) 
8. Produce the new solutions vi,j for the onlookers from the solutions xi,j selected depending on $\mathrm{Pi}, \mathrm{j}$ and evaluate them

9. Apply the greedy selection process

10. Determine the abandoned solution for the scout, if exists, and replace it with a new randomly produced solution $x i, j$

11. Memorize the best solution achieved so far

12. cycle $=$ cycle +1

13. until cycle $=\mathrm{MCN}$

Figure 1 represents a flowchart for simple Artificial Bee Colony (ABC) algorithm. According to this algorithm an initial path is created which goes directly from the starting point to the target and turns if an obstacle is encountered. The length of the path is calculated in the next step [10]. The employed bees are then sent to the neighbourhood of breakpoints and the fitness of the new paths is calculated. In the next step the onlooker bees are sent to the selected breakpoint of the neighbourhood for exploitation. The fitness function is then calculated for new paths. In the next step the scout bees are sent for exploration and for finding new paths. The above steps are repeated until the most optimum path is found.



Figure 1: Flowchart for ABC Algorithm

\section{COMPARISON BETWEEN ACO AND ABC FOR PATH OPTIMIZATION}

Both Ant colony Optimization (ACO) and Artificial Bee Colony (ABC) optimization algorithm can be used for optimizing the path traversed. Each of these algorithms has its own advantages and disadvantages and thus can be used for different purposes depending upon the need of the situation.

This section explains the basic concept behind the ACO algorithm and the compares $\mathrm{ACO}$ and $\mathrm{ABC}$ and presents advantages and disadvantages of both.

\subsection{Ant Colony Optimization}

Ant Colony Optimization algorithm was inspired by one the most famous biological experiments named as "Double Bridge Experiment".

In the experiment a double bridge with two branches of varying lengths connected the nest of this species with a food source. The long branch of the bridge was twice as long as the shorter branch. In most runs of this experiment it was observed that after certain interval of time nearly all ants use the shorter branch [11].

The explanation of this behaviour is based on the fact that the ants lay pheromone along their path. Ants which randomly choose the shorter branch arrive earlier at the food source. When they go back they smell some pheromone on the shorter branch and hence prefer this branch. As a result, the pheromone on the shorter branch accumulates faster than on the longer branch. Due to increasing concentration of pheromone on the former branch all ants prefer this branch. Similar to the experiment with branches of different lengths, when both branches have the same length; it is observed that after certain time interval nearly all ants use the same branch [12]. But in several repetitions it is a random process which of the two branches will be chosen. The reason for this is that when one branch has got a slightly higher pheromone concentration due to random fluctuations that particular branch will be preferred by the ants so that the difference in pheromone concentration will increase and after some time all ants will take this branch.

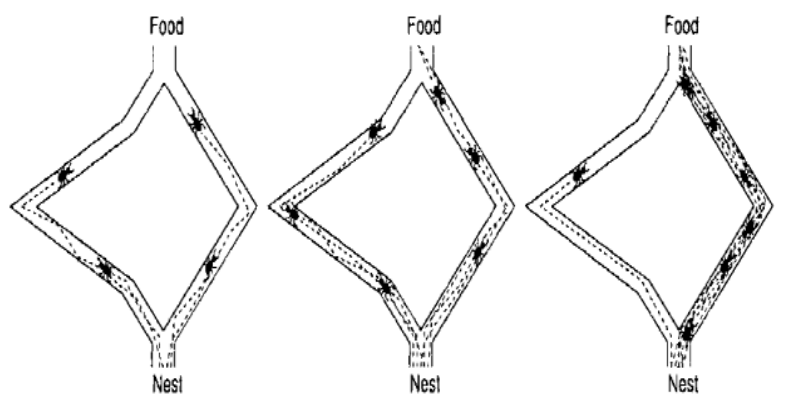

Figure 2: Double Bridge Experiment

\subsection{Pseudo Code for ACO}

The pseudo code for Ant colony Optimization algorithm can be defined as follows:

initialize all edges to (small) initial pheromone level $\tau 0$; place each ant on a randomly chosen city; for each iteration do:

do while each ant has not completed its tour: for each ant do: move ant to next city by the probability 
end;

for each ant with a complete tour do:

evaporate pheromones;

apply pheromone update;

if (ant k's tour is shorter than the global

solution)

update global solution to ant k's tour

end;

the flowchart for the same is as shown in figure 3.

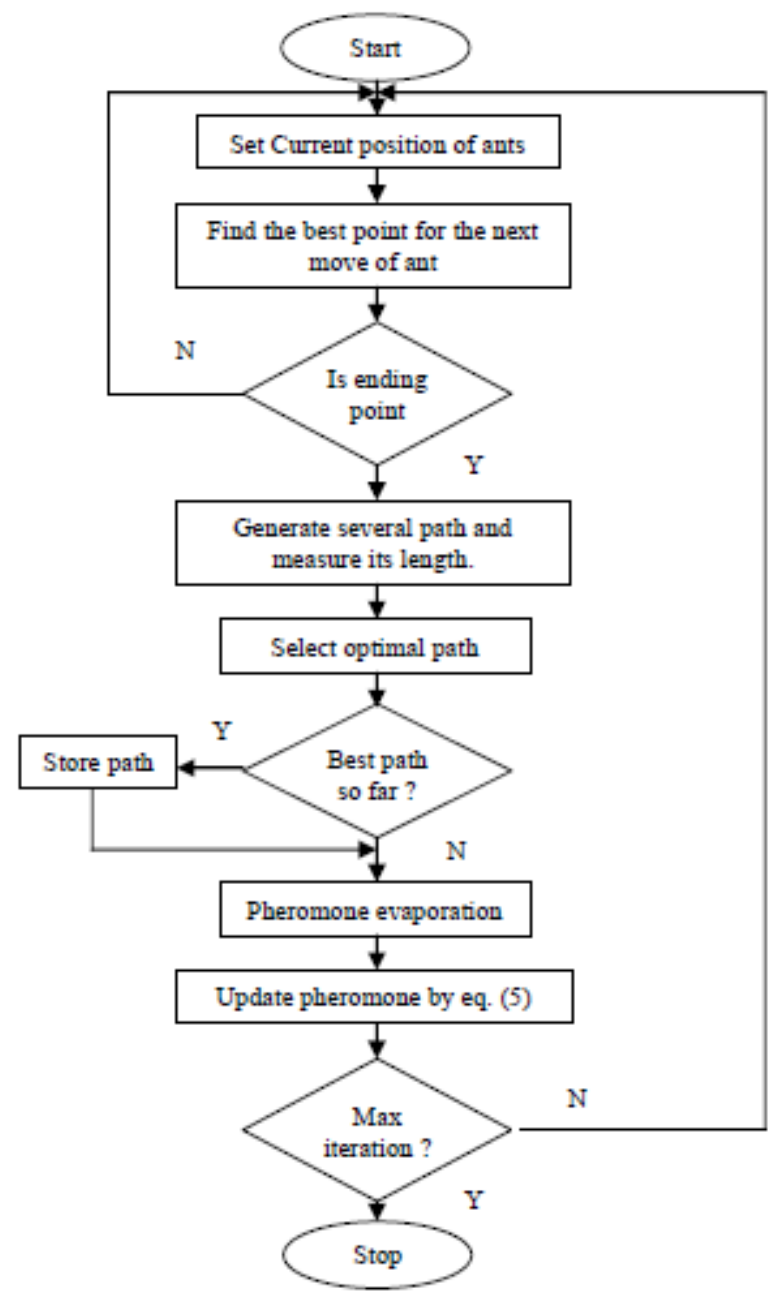

Figure 3: Flowchart for ACO

\subsection{Comparison}

Ant colony Optimization algorithm has been inspired by the behaviour of ant colonies and based on Pheromone trails. Whereas, the Artificial Bee Colony algorithm was inspired by collective behaviour of honey bees and based on mysterious dance inside the hives [13].

ACO is mostly applied to discrete combinatorial optimization problems. On the other hand, $\mathrm{ABC}$ is applied to Constraint and Unconstraint Optimization problems [14].

The solution is represented by the path constructed by the ants and by the position of bee's in the neighbourhood of breakpoint for $\mathrm{ACO}$ and $\mathrm{ABC}$ respectively.

The knowledge in the case of ACO is stored in the pheromone levels associated with the path trails. Whereas, for $\mathrm{ABC}$, knowledge is stored in the dance which is performed by employee bees [15].

\section{CONCLUSION}

In this paper, we have highlighted the need of path optimization. Due to increasing time constraints, there is a need to cover the path in the most optimum manner. Where, the path is defined as the trajectory to be followed starting from a specific start position up to a predefined destination or goal point. Further, we have described Artificial Bee Colony Algorithm, which is one of the many techniques of Swarm Intelligence and can be successfully applied for optimizing the path. Moreover, a comparison between two Swarm Intelligence techniques, namely Artificial Bee Colony algorithm and Ant Colony Optimization algorithm is presented. It is observed that ants are good in search and exploitation and thus ACO can be used for dynamic applications. However, its theoretical analysis is difficult and the probability distribution changes by iteration. Moreover, the time for convergence is uncertain. $\mathrm{ABC}$ on the other hand, employees fewer parameters, it has strong robustness, fast convergence and high flexibility. It can also be used for solving multimodal and multidimensional optimization problems. In addition to this, $\mathrm{ABC}$ has global optimization and easy recognition. It conducts both local search and global search in each iteration and as a result the probability of finding the optimal increases. The structure of the $\mathrm{ABC}$ algorithm is such that it supports parallel processing as result saving time. Considering the wide number of advantages of Artificial Bee Colony algorithm (ABC) in comparison to other Swarm intelligence techniques, it can be concluded that $\mathrm{ABC}$ is the most efficient algorithm for optimizing a given path.

\section{REFERENCES}

[1] Bonabeau, E., Dorigo, M., \& Theraulaz, G. (1999). Swarm intelligence (pp. 32-77). Oxford.

[2] Sierakowski, Cezar A., and Leandro dos S. Coelho. "Study of two swarm intelligence techniques for path planning of mobile robots." 16th IFAC World Congress, Prague. 2005.

[3] Dorigo, Marco, Gianni Di Caro, and Luca M. Gambardella. "Ant algorithms for discrete optimization." Artificial life 5.2 (1999): 137-172.

[4] Yang, Xin-She. "Firefly algorithms for multimodal optimization." Stochastic algorithms: foundations and applications. Springer Berlin Heidelberg, 2009. 169-178.

[5] Wang, Kang-Ping, et al. "Particle swarm optimization for traveling salesman problem." Machine Learning and Cybernetics, 2003 International Conference on. Vol. 3. IEEE, 2003.

[6] Teodorović, Dušan, and Mauro Dell'Orco. "Bee colony optimization-a cooperative learning approach to complex transportation problems." Advanced OR and AI Methods in Transportation: Proceedings of 16th Mini-EURO Conference and 10th Meeting of EWGT (13-16 September 2005).-Poznan: Publishing House of the Polish Operational and System Research. 2005.

[7] Ahmed, Hazem, and Janice Glasgow. "Swarm Intelligence: Concepts, Models and Applications." School Of Computing, Queens University Technical Report(2012).

[8] Saffari, M. H., and M. J. Mahjoob. "Bee colony algorithm for real-time optimal path planning of mobile robots." Soft Computing, Computing with Words and Perceptions in 
System Analysis, Decision and Control, 2009. ICSCCW 2009. Fifth International Conference on. IEEE, 2009.

[9] Karaboga, Dervis, and Bahriye Basturk. "Artificial bee colony (ABC) optimization algorithm for solving constrained optimization problems."Foundations of Fuzzy Logic and Soft Computing. Springer Berlin Heidelberg, 2007. 789-798.

[10] Lin, Jiann-Horng, and Li-Ren Huang. "Chaotic bee swarm optimization algorithm for path planning of mobile robots." Proceedings of the 10th WSEAS international conference on evolutionary computing. World Scientific and Engineering Academy and Society (WSEAS), 2009.

[11] Dorigo, Marco, and Thomas Stützle. "Ant colony optimization: overview and recent advances." Handbook of metaheuristics. Springer US, 2010. 227-263.
[12] Pal, Narendra Singh, and Sanjeev Sharma. "Robot Path Planning using Swarm Intelligence: A Survey." METHODS 83.12 (2013).

[13] Oliveira, IMS de, Roberto Schirru, and J. A. C. C Medeiros. "On the Performance of an Artificial Bee Colony Optimization Algorithm applied to the accident diagnosis in a PWR Nuclear Power Plant." International Nuclear Atlantic Conference. 2009.

[14] Dorigo, Marco, and Mauro Birattari. "Ant colony optimization." Encyclopedia of Machine Learning. Springer US, 2010. 36-39.

[15] Karaboga, Dervis, and Bahriye Basturk. "On the performance of artificial bee colony (ABC) algorithm." Applied soft computing 8.1 (2008): 687-697. 